\title{
Study on Generation Mechanism of Abnormal Vibration of Flow Dynamic Conveyer*
}

\author{
Kunihiko ISHIHARA** and Daisuke ETO** \\ **Institute of Technology and Science, The University of Tokushima \\ 2-1 Minami-Jyosanjima-Cho, Tokushima-City, Tokushima-Pref. 770-8506, Japan
}

\begin{abstract}
The FDC (Flow Dynamics Conveyer) has often been used in power plants and iron works because of its superiority in low noise and power compared with roller type conveyers. The FDC consists of a trough and a belt, and the air is supplied from a series of holes provided on the trough. However, large vibrations occur when the flow rate reaches a certain value. This abnormal vibration is defined to be self-excited vibration caused by leakage flow. In this study, as a first step, the theoretical model of a non-tapered clearance flow passage is examined and the natural frequency is calculated. In addition, experiments are conducted to examine the validity of the theory. As a second step, in the case of a tapered clearance flow passage, a concentrated load is added to the belt. The effects of the belt deformation, the flow rate, and the added loads on the clearance flow are examined along with considering the generation mechanism of the abnormal vibration. As a result, it was clarified that the generation mechanism of the self-extracted vibration was due to the phase shift by a change in parameters.
\end{abstract}

Key words: Flow Dynamic Conveyer, Natural Frequency, Self-Excited Vibration, Leakage Flow

\section{Introduction}

The Flow Dynamic Conveyor (FDC) has been used more than the conventional roller conveyer due to its advantages of quiet low power and high speed. However, abnormal vibrations sometimes occur during certain operating conditions, such as when the load and flow rate of supplied air are wrong by design and manufacture. The abnormal vibration causes a scaffolding quake and noise, and resulting in the need to halt operations. The cause of the abnormal vibration can be considered to be the leakage flow between the trough and the belt.

In past studies, Inada and Hayama $[1,2]$ evaluated the fluid dynamic forces generated in parallel vibrating of one-dimensional tapered clearances. While assumptions still exist, the results are useful in understanding the phenomenon due to an analytical approach. Subsequently, Arai et al. [3] studied the vibrations of asymmetric bodies caused by leakage flow analytically, and $\mathrm{Li}$ et al. [4,5] studied the leakage flow of an annular clearance.

However, the natural frequency and the stability of a real FDC cannot be discussed due to the lack of a gravity effect on the fluid force in these studies. On the contrary, in a recent study, Omori et al. [6,7] studied the stability of the FDC considering the gravity effect, and Ishihara $[8,9]$ considered the phenomenon of abnormal vibration of real FDC by measuring the vibration and pressure. However, still unclear is how the natural frequency of the FDC is determined.

Therefore, the purpose of this study is to clarify how the natural frequency of the FDC is determined, and to obtain an area of instability of a FDC. First, a theoretical model of a non-tapered clearance flow passage is supposed and the natural frequency is calculated. In 
the theory, the relationship between the loads and the clearance with parameters of the flow rate is obtained. The air stiffness from the flow can be obtained by differentiating the expression with the clearance, and the natural frequency can be calculated by using the stiffness and the loaded mass. Based on those calculations, experiments are conducted and the validity of the theory is examined. Secondly, in the case of a tapered clearance flow passage, the concentrated load is added to the belt, and the effects of the belt deformation, flow rates, and added loads on the clearance flow are examined. In addition, the generation mechanism of the abnormal vibration is considered. In order to clarify the generation mechanism of the abnormal vibration, the vibration (displacement) and the fluctuating pressure in the flow channel made by the trough and the belt were measured simultaneously.

\section{Theory}

\subsection{Theoretical Model}

Fig.1 shows the cross-section of a FDC. The belt runs on a circular trough and its weight is supported by the air supplied between the belt and the trough. Fig.2 shows the theoretical model. This model corresponds to the right half of the Fig. 1 as shown by the dotted line in this figure.

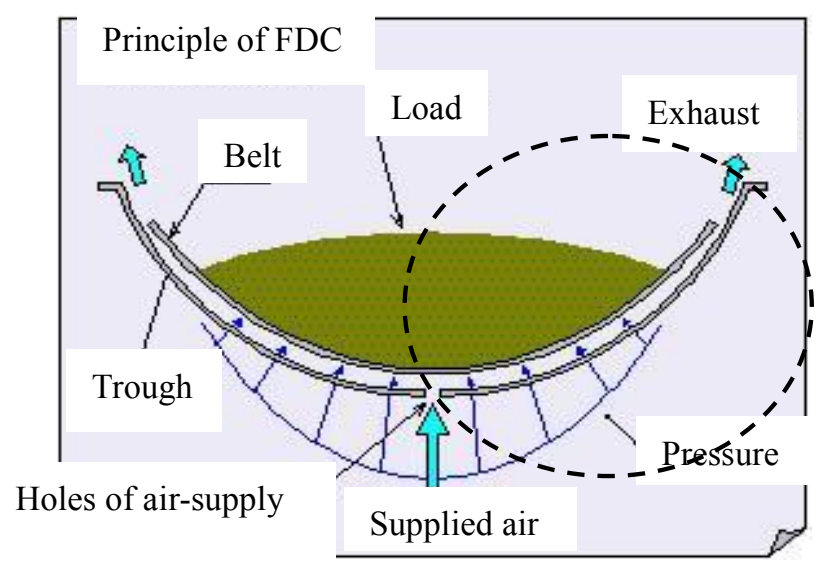

Fig. 1 Cross section of FDC

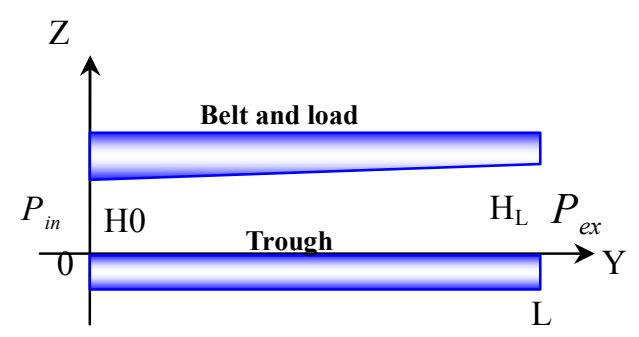

Fig.2 Analytical model

The belt is assumed to be a tapered plate. The cross-section has a unit-length in depth and is considered two-dimensional. The belt and trough are assumed to be rigid bodies and the belt moves only in the $\mathrm{Z}$ direction. The trough is considered to be a stationary wall. The air flow is regarded as non-compressible and viscous. 


\subsection{Equations}

The flow rate $Q\left(\mathrm{~m}^{3} / \mathrm{min} / \mathrm{m}\right)$ and the steady fluid force $F(\mathrm{~N} / \mathrm{m})$, according to Inada and Hayama [1], are described as follows.

$$
\begin{gathered}
\frac{\bar{Q}}{H_{0}}=\sqrt{\frac{P_{i n}-P_{e x}}{\rho\left\{(\beta-\alpha) I_{3}(1)+\frac{\xi_{i n}}{2}+\frac{\xi_{e x}}{2 h^{2}(1)}\right\}}} \\
\frac{\bar{F}}{L P^{*}}=\frac{P_{\text {in }}}{P^{*}}-\frac{\xi_{\text {in }}}{2}-\frac{(\beta-\alpha)}{2(1+\alpha)}
\end{gathered}
$$

Where $\alpha$ is the channel enlargement factor and is defined as $\alpha=\left(H_{L}-H_{0}\right) / H_{0}$. $\xi_{\text {in }}$ and $\xi_{\text {out }}$ are the inlet and the outlet loss factors respectively. The other parameters are given as follows.

$$
\left.\begin{array}{l}
{ }^{*}=\frac{\rho \overline{Q^{2}}}{H_{0}^{2}} \\
\beta=\frac{\bar{\lambda} L}{4 H_{0}}, \\
I_{3}(y)=\int_{0}^{y} \frac{d y}{h^{3}}=\frac{1}{2 \alpha}\left(1-\frac{1}{h^{2}}\right) \\
\bar{\lambda}=\frac{48 \cdot v}{\bar{Q}}
\end{array}\right\}
$$

Eliminating $P_{\text {in }}$ from these two equations and organizing them, Eq.(4) can be obtained and it is expressed as a third-order algebraic equation of $H_{0}$

$$
\left(\frac{\bar{F}}{L}-P_{e x}\right) \cdot H_{0}^{3}-\rho \bar{Q}^{2} \cdot\left\{\frac{\xi_{e x}}{2 h^{2}(1)}-A \cdot \alpha\right\} \cdot H_{0}-\rho \bar{Q}^{2} A \cdot \frac{12 L v}{\bar{Q}}=0
$$

in which $A=I_{3}(1)-\frac{1}{2(1+\alpha)}$.

Generally this vibration system is considered to be a nonlinear system. However, the belt vibrates with small amplitude around an average elevation. Then, the vibration system is considered to be a linear system and the effective stiffness can be obtained by the gradient of the $F-H_{0}$ curve at average elevation $H_{0}{ }^{*}$. When the flow rate changes, the average elevation of $H_{0}{ }^{*}$ changes, as well. As a result the effective stiffness $k$ also changes. In this study, it is considered that the gradient of the $F-H_{0}$ curve is locally interpreted as a spring constant of air flow. Differentiating the steady fluid force $\bar{F}$ with $H_{0}$, Eq. (5) can be obtained.

$$
k=\frac{d \bar{F}}{d H_{0}}=\rho \bar{Q}^{2} L \cdot\left(\xi_{\text {in }}-\frac{\alpha}{1+\alpha}\right) \cdot H_{0}^{-3}+\frac{18 v L^{2} \rho \bar{Q}}{(1+\alpha)} \cdot H_{0}^{-4}
$$

$M$ being the sum of the masses of the belt and the load, the natural frequency becomes Eq. (6)

$$
f_{n}=\frac{1}{2 \pi} \cdot \sqrt{\frac{k}{M}}
$$

where $L$ is the belt length (m). $P_{\text {in }}$ and $P_{\text {out }}$ are the inlet and outlet pressures $(\mathrm{Pa})$ respectively. $\rho$ is the air density $\left(\mathrm{kg} / \mathrm{m}^{3}\right), v$ is the kinetic viscosity $\left(\mathrm{m}^{2} / \mathrm{s}\right)$, and $\xi_{\text {in }}$ and $\xi_{\text {out }}$ are the inlet and outlet loss factors, respectively. $\alpha$ is an enlargement factor, $h$ is the non dimensional steady clearance, and $\lambda$ is the friction factor.

\subsection{Calculation Conditions}

The relation between the steady clearance $H_{0}$ and load $W$ (=belt + load) is examined by using the Eq. (4). It is assumed here that the steady force $F$ is in equilibrium with the load. 
First of all, each parameter is applied to Eq. (1) and the third-order algebraic equation is solved. The real solution of these roots is the steady clearance. The flow rates are changed to $1.27,1.14,1.00,0.846,0.643 \mathrm{~m}^{3} / \mathrm{min} / \mathrm{m}$, and the loads are changed to $12,9,6,3$, $0 \mathrm{~kg}$ by piling the plate one by one for each flow rate.

By giving steady clearances obtained by the calculation and each parameter to Eq. (5), the air spring constant can be calculated. As a result, the natural frequency can also be obtained. The calculation is carried out under the following conditions. The mass of belt $=2.3 \mathrm{~kg}, \xi_{\text {in }}=0.25, \xi_{\text {ex }}=1.0, P_{\text {ex }}=0 \mathrm{~Pa}, \quad L=0.250 \mathrm{~m}$ and $\rho=1.2 \mathrm{~kg} / \mathrm{m}^{3}$

\section{Experiment (In the Case of a Flow Channel without a Taper)}

\subsection{Experimental Apparatus}

Fig. 3 shows the experimental apparatus of the FDC model. The belt and trough are made from stainless steel. The duct and the blower are connected by a PVC pipe. The belt is $500 \mathrm{~mm} \times 500 \mathrm{~mm} \times 1.2 \mathrm{mmt}(\mathrm{t}$ :thickness) and $2.3 \mathrm{~kg}$. Iron plates of $484 \mathrm{~mm} \times 484 \mathrm{~mm} \times 1.5$ $\mathrm{mm}(3.0 \mathrm{~kg})$ are used as loads in order to send a uniform amount of loads to the belt. The ring blower is used to give the air flow to the FDC. The flow rate is controlled by a ball valve. A $\phi 1 \mathrm{~mm}$ hole is opened to the trough. The hole and the digital manometer are connected by a tube to measure the pressure between the belt and trough

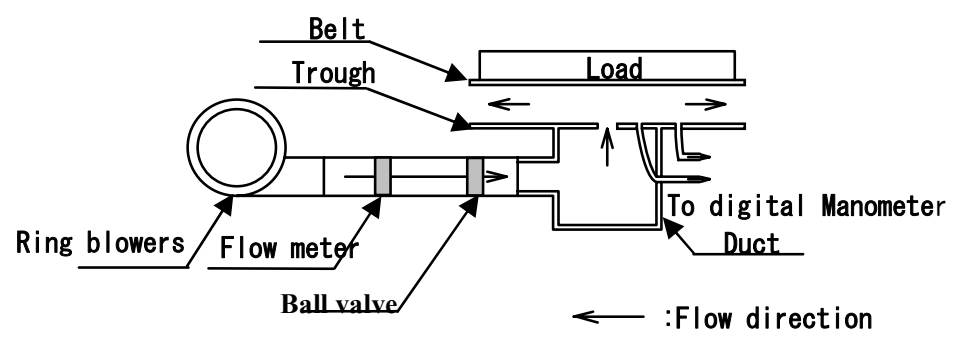

Fig.3 Experimental apparatus

\subsection{Experimental Procedure}

The natural frequency of a FDC in operation is obtained by a hammering test. In addition, the floating displacements are measured at five points as shown in Fig. 4 by the laser displacement sensor and averaged.

\subsection{Experimental Results and Considerations}

Fig. 5 shows the relation between the steady clearance $H_{0}$ and the load $W$ at each flow rate $Q$. The solid lines show the theoretical values and these are the results under the calculation conditions $\xi_{\mathrm{in}}=0.25, \xi_{\mathrm{ex}}=1.0$, and $P_{e x}=0 \mathrm{~Pa}$. The theoretical results of $W$ and $H_{0}$ are in good agreement with the experimental ones. However, the theoretical values of $H_{0}$ in the case of $W=22.6 \mathrm{~N}$ (No Load) are $7 \sim 14$ percent less than the experimental values. In order to examine the cause, the deflection of the belt was measured. As a result, the deflection of the belt was deformed to a convex as shown in Fig.6. The flow channel becomes smaller from the inlet to the outlet resulting in pressurization.

Fig.7 shows the results of the time history and the frequency analysis of the belt displacement under the condition of $W=9 \mathrm{~kg}$ and $Q=1.27 \mathrm{~m}^{3} / \mathrm{min} / \mathrm{m}$. As can be seen from the result, the belt vibration damps after the initial impact and the natural frequency is $26.25 \mathrm{~Hz}$. Fig. 8 shows the relation between the natural frequency and the load as the parameter of $Q$. The theoretical results indicated by several lines show that the natural frequency becomes smaller with an increasing flow rate. On the contrary, the experimental results indicated by 
symbols show that the natural frequency is almost independent of the flow rate. The cause of the difference has not been clear in the present stage.

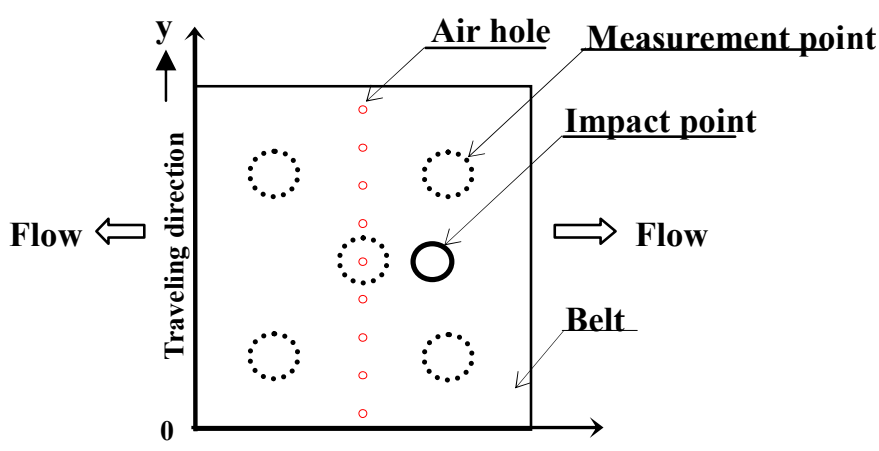

Fig.4 Measurement points of belt

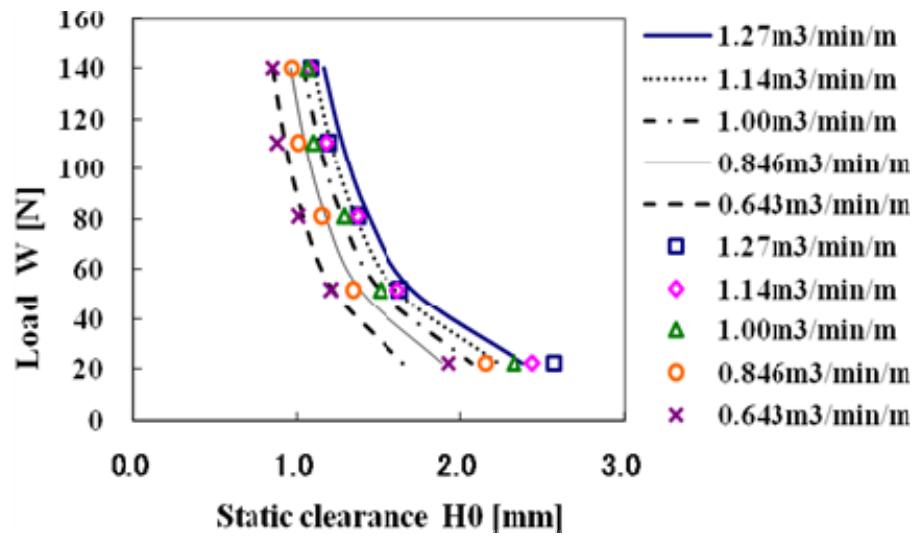

Fig.5 Relation between static clearance and load

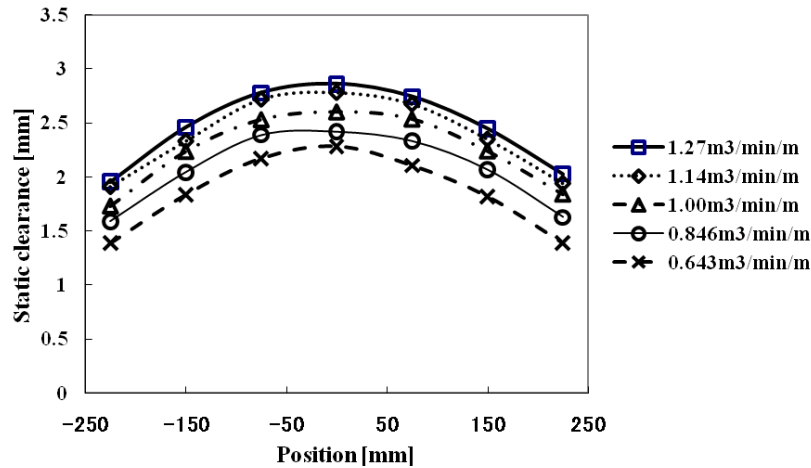

Fig.6 Deflection of the belt 

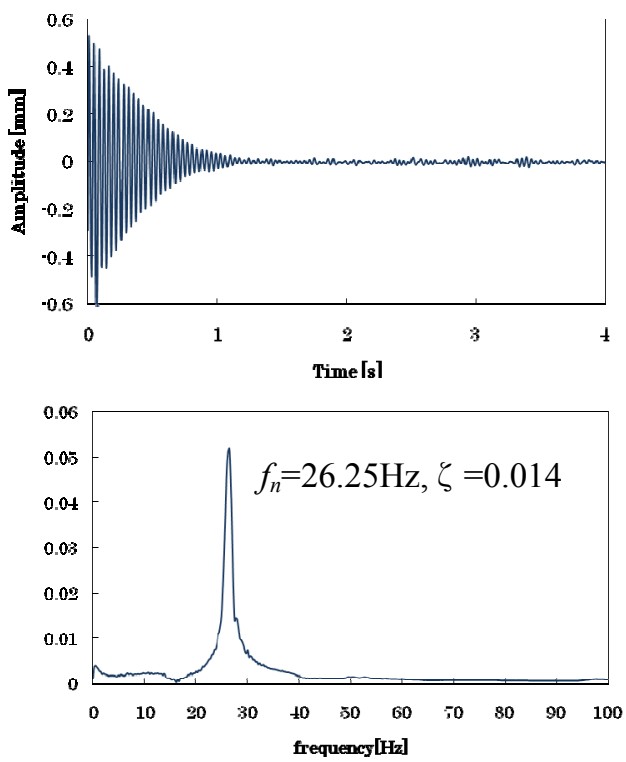

Fig. 7 Time waveform and spectral graph of $9 \mathrm{~kg}$ and $1.27 \mathrm{~m}^{3} / \mathrm{min} / \mathrm{m}$

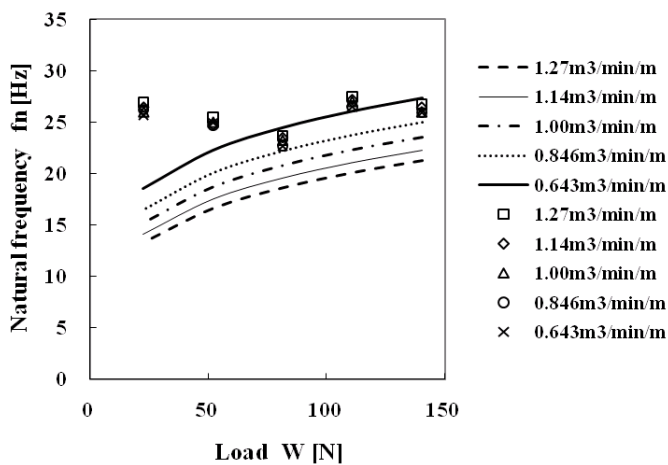

Fig.8 Relation between load and natural frequency

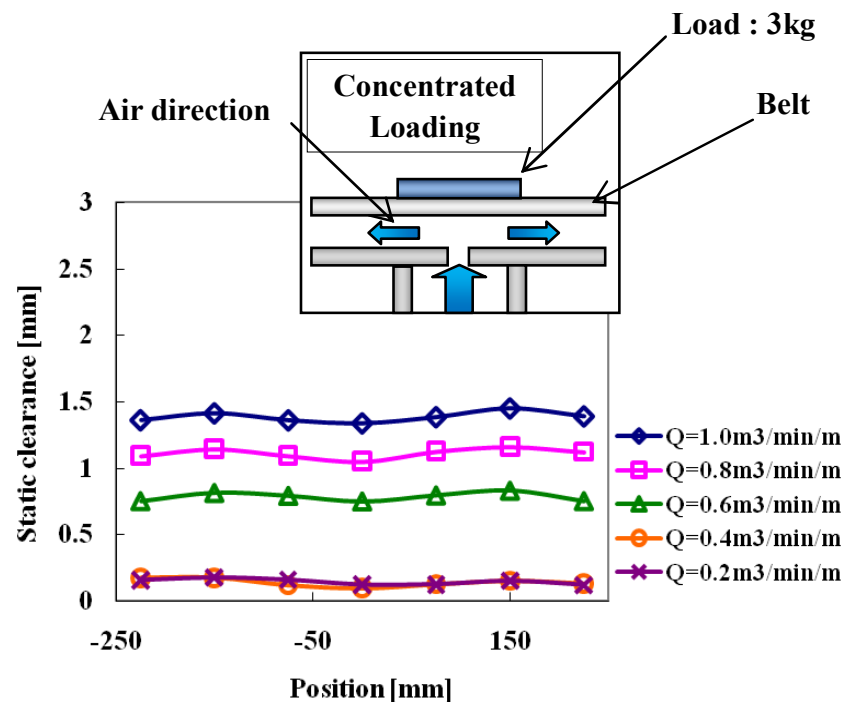

Fig.9 Belt deflection 
Next, the experimental result of the natural frequency does not have the tendency similar to the theoretical one in the case of $W=22.6 \mathrm{~N}$ (No Load) and $W=52.0 \mathrm{~N}$.

As is seen from Fig.6, the belt was deformed in a convex in the cases of these loads. This contracts the flow channel. Consequently, the pressure between the trough and belt rises up with the outlet resistance becoming large from the performance curve of the blower. This pressurization brings the larger stiffness $k$ and a higher natural frequency.

Fig. 9 shows the steady clearance when the concentrated load (weight $=3 \mathrm{~kg}$ and the size: $443 \mathrm{~mm} \times 100 \mathrm{~mm} \times 9 \mathrm{mmt}$, t:thickness) is put on the center of the belt as shown in Fig.9.

The belt deformation disappears and it can be regarded as a parallel channel. That is to say, the flow channel is supposed to be the contracted channel in the case of applying the uniform load of $3 \mathrm{~kg}$. From this, it can be considered that the experimental result of the natural frequency became larger than the theoretical one in the case of $0 \mathrm{~kg}$ and $3 \mathrm{~kg}$ loads.

\subsection{Proof of Presumption}

In order to prove the presumption described above, the natural frequency and the steady clearance are measured under the condition of no deformation by infixing the rigidity stick on the belt at the position indicated by a dashed line in Fig.4; as shown in Fig.10. The rigidity stick is made of aluminum and is $490 \mathrm{~mm}$ and $385 \mathrm{~g}$.

Fig. 11 and Fig 12 show the steady clearance before and after the infixing, respectively.

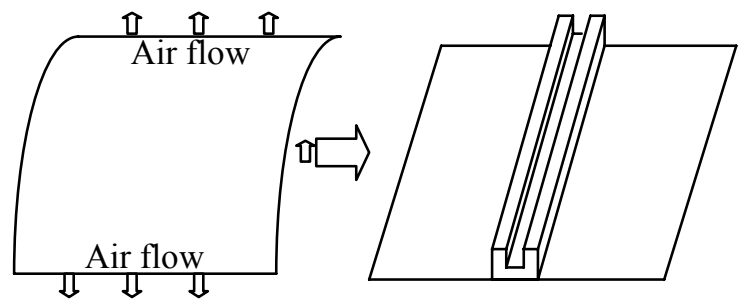

Fig.10 Installation of rigidity stick

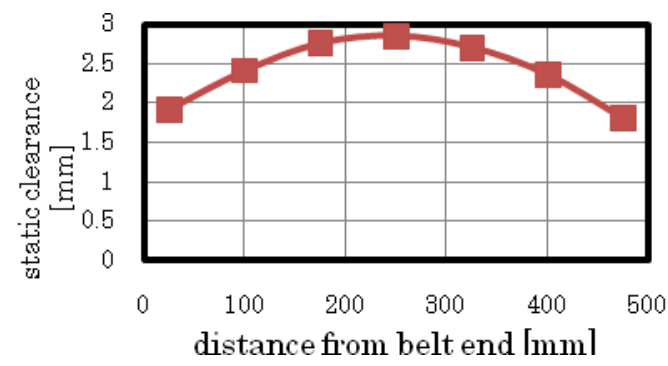

Fig.11 Static clearance (without rigidity stick)

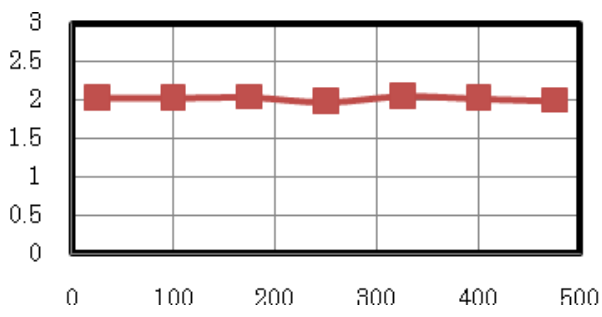

Fig.12 Static clearance (with rigidity stick) 
As can be seen from these figures, the deformation of the belt disappears by infixing the rigidity stick on the belt. The rigid body is assumed in the theory. The uniformity of belt deformation in the travelling direction was confirmed.

Fig.13 and Fig.14 show the experimental results of the relation between the load and the natural frequency, and the relation between the static clearance and the load, respectively. The solid lines show the theoretical results and the symbols show the experimental results. The natural frequency of the belt approached the theoretical results by reducing $26 \mathrm{~Hz}$ to $21.5 \mathrm{~Hz}$ in the case of $W=22.6 \mathrm{~N}$. The steady clearance also approached the theoretical result compared with the result when the deformation of the belt exists. This supports the presumption described in section 3.3, and it was clarified that the difference between the theoretical and experimental results appeared due to the deformation.

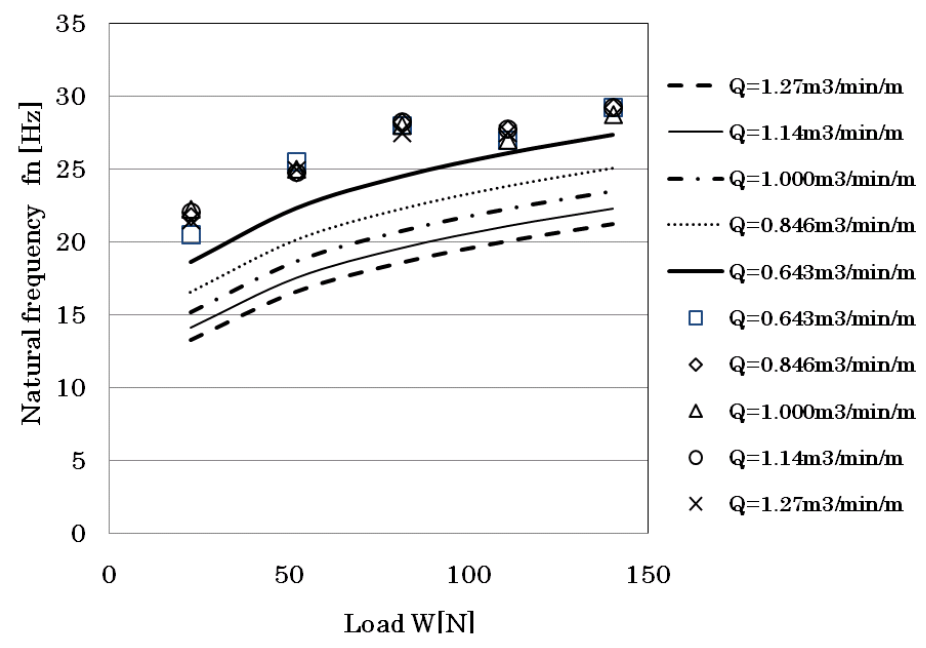

Fig.13 Relation between load and natural frequency after using rigidity stick

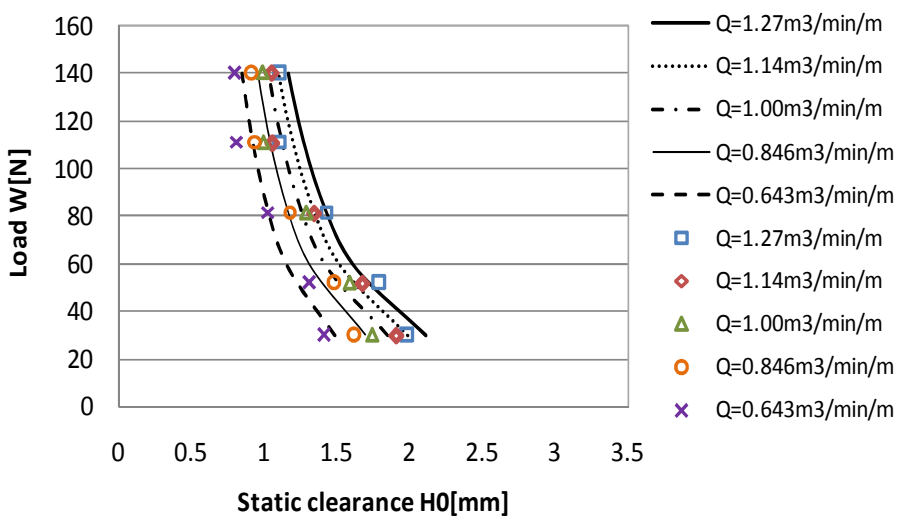

Fig.14 Relation between static clearance and load after using rigidity stick

\section{Experiment (In the Case of a Flow Channel with Taper)}

\subsection{Experimental Apparatus}

The experimental apparatus is identical to the one in Fig.3. The ring blower is used with a constant rotating speed. The air compressed by the ring blower passes in a pipe and the flow rate is measured by the flow meter. The flow meter consists of a Venturi tube and a liquid-level manometer. The flow rate is adjusted by the ball valve. The compressed air enters the duct after adjusting the flow and enters between the trough and belt from many 
holes opened on the trough and supports the belt. The elevation of the belt is measured by the laser displacement fixed on the traverse equipment as shown in Fig.15, and the data is recorded with the memory card. Iron plates $(443 \mathrm{~mm} \times 100 \mathrm{~mm} \times 9 \mathrm{mmt}$, $\mathrm{t}$ : thickness $)$ are used as concentrated loads. The plates were $3 \mathrm{~kg}$ each and were piled in the center of the belt. By doing this, the belt deforms as a convex and a divergent flow channel is formed. This becomes the generating equipment of abnormal vibration.

\subsection{Generation Region of Abnormal Vibration}

The concentrated load is used as the load and the weight is changed. Nine kinds of loads $(0,3,6,7,8,9,10,11,12 \mathrm{~kg})$ were used as experimental parameters and some flow rates. The flow rate was changed from low to high values by the ball valve at each load and then decreased. By doing this, the flow rate at the beginning and the end of the abnormal vibration could be obtained. The amplitude of belt at the abnormal vibration is measured at a combination of various flow rates and loads in order to obtain the generation region of the abnormal vibration. This measurement was carried out at the position indicated by the circle shown in Fig.16.

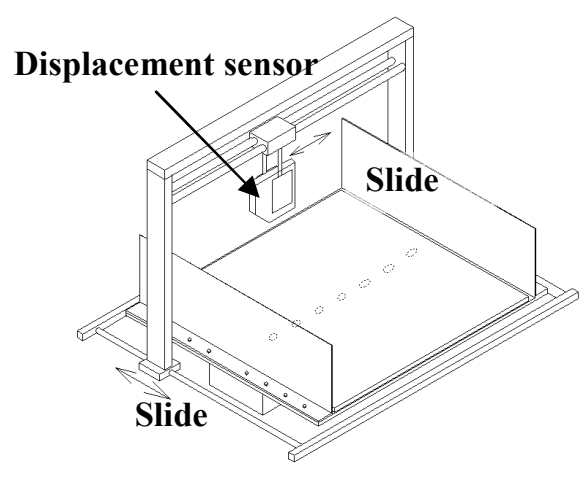

Fig.15 Traverse equipment

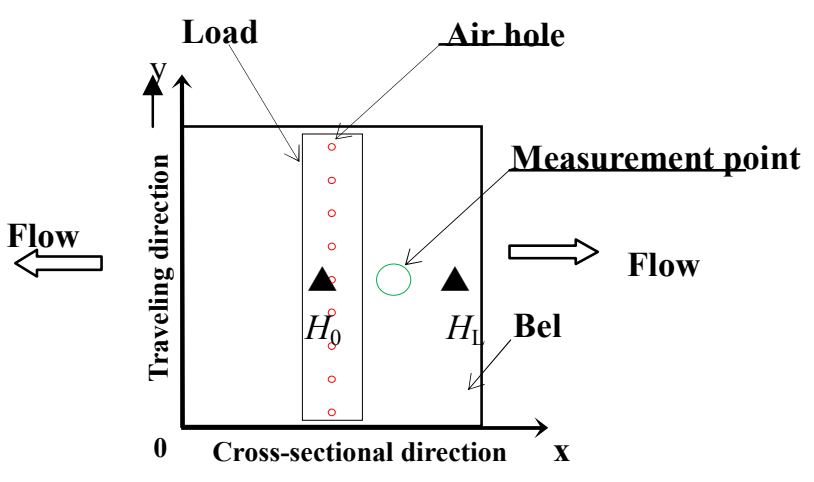

Fig.16 Measurement Point of unusual vibration

\subsection{Experimental Results and Discussion}

Fig. 17 and 18 are the time histories and the frequency spectra of the belt vibration after impact at $Q=0.8 \mathrm{~m}^{3} / \mathrm{min} / \mathrm{m}$ and $Q=0.4 \mathrm{~m}^{3} / \mathrm{min} / \mathrm{m}$, respectively, when the concentrated load $\mathrm{ml}$ was $7 \mathrm{~kg}$. The former is the result of stable and the latter is the result of unstable conditions. The limit-cycle forms in the latter case.

Fig.19 and 20 show the stability map based on the flow rate $Q$ and the load $W$. Half the amplitude is shown by the radius of the circle. The graph legends " $Q_{u p}$ " and " $Q$ down" 
mean the increasing and decreasing flow rates, respectively. The suffixes "start-up" and "end" show the beginning and the end of the abnormal vibration, respectively. The performance of the limited ring blower resulting in the flow rate limit is shown in Fig.19. The abnormal vibration does not occur from $0 \mathrm{~kg}$ to $6 \mathrm{~kg}$ independent of the flow rate.
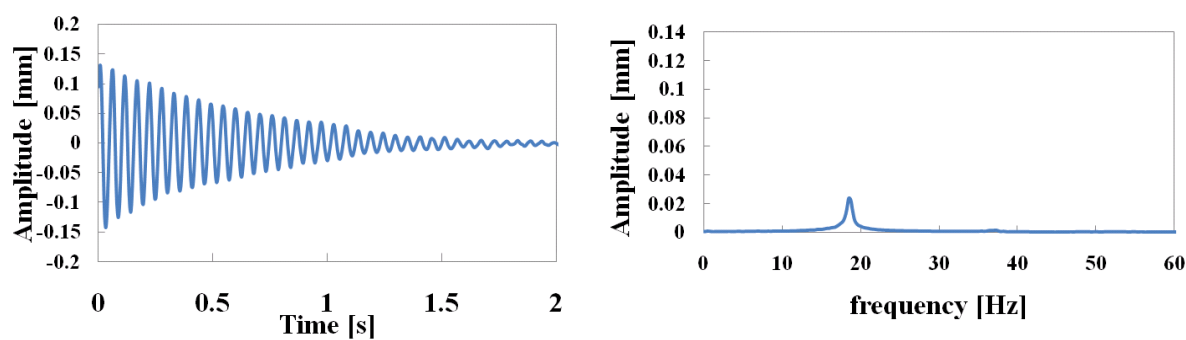

Fig.17 Time history and spectrum of damped vibration $\left(Q=0.8 \mathrm{~m}^{3} / \mathrm{min} / \mathrm{m}\right)$
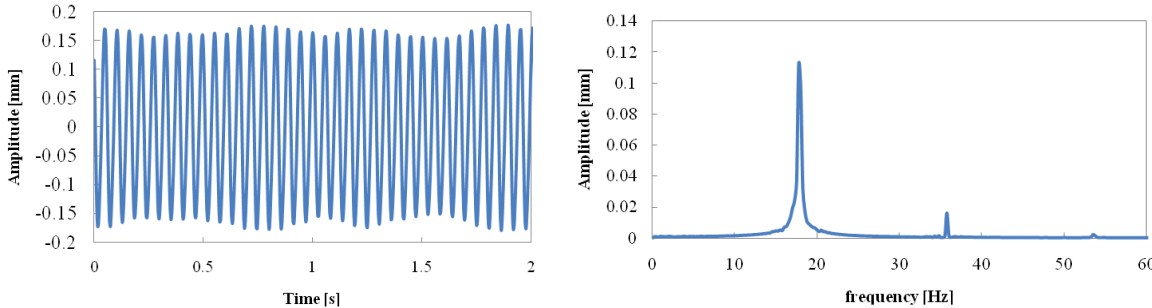

Fig.18 Time History and spectrum of abnormal vibration $\left(Q=0.4 \mathrm{~m}^{3} / \mathrm{min} / \mathrm{m}\right)$

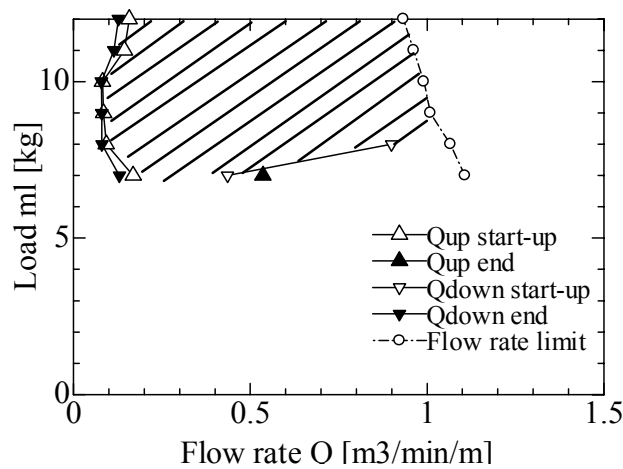

Fig.19 Unusual vibration generation area

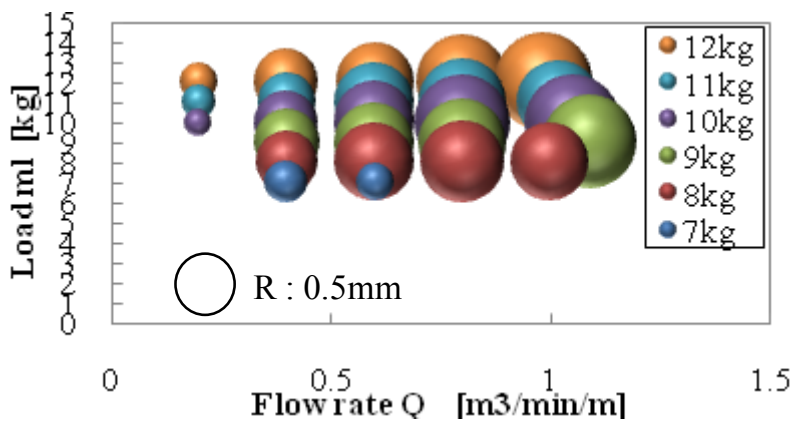

Fig.20 Stable map 
This is because the divergent flow channel needed for the abnormal vibration to occur was not formed. Fig. 21 shows the relation between the load $\mathrm{ml}$ and the enlargement factor $\alpha$ as the parameter of the flow rate $Q . \quad \alpha$ is obtained as $\alpha=\left(H_{\mathrm{L}}-H_{0}\right) / H_{0}$. Where $H_{0}$ and $H_{\mathrm{L}}$ are measured at the position of $\boldsymbol{\Delta}$ in Fig.16. $\alpha$ is the same value independent of $Q$. There is a tendency that $\alpha$ increases with load $m l$ and suddenly increases at $m l=7 \mathrm{~kg}$ which is the weight when the abnormal vibration begins to occur. This becomes a constant value (1.0) of over $m l=8 \mathrm{~kg}$ which is the weight when the abnormal vibration occurs perfectly. The reason has not been clear now, but it is a very interesting result. We want to study it in the near

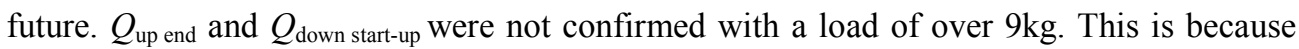
$Q_{\text {up end }}$ and $Q_{\text {down start-up }}$ exist in the flow region over the ring blower performance. From this result, it was clarified that the flow region generating the abnormal vibration becomes wider with an increase in the load.

Fig.22 shows the time histories of the belt deflection and the fluctuation pressure between the trough and belt. The upper and lower figures show the results of $Q=0.2 \mathrm{~m}^{3} / \mathrm{min} / \mathrm{m}$ and $Q=0.8 \mathrm{~m}^{3} / \mathrm{min} / \mathrm{m}$ in the case of a $12 \mathrm{~kg}$ load, respectively.

Fig.23 shows the lissajous orbits of the pressure fluctuation and the belt deflection corresponding to Fig. 22 .

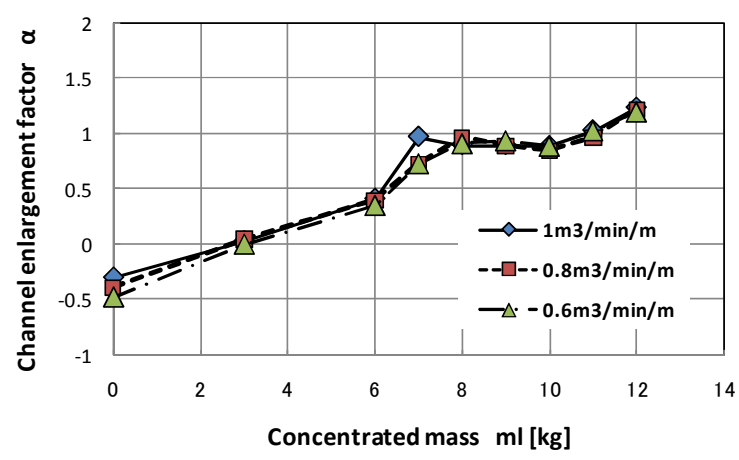

Fig.21 Relation between $\alpha$ and $m l$
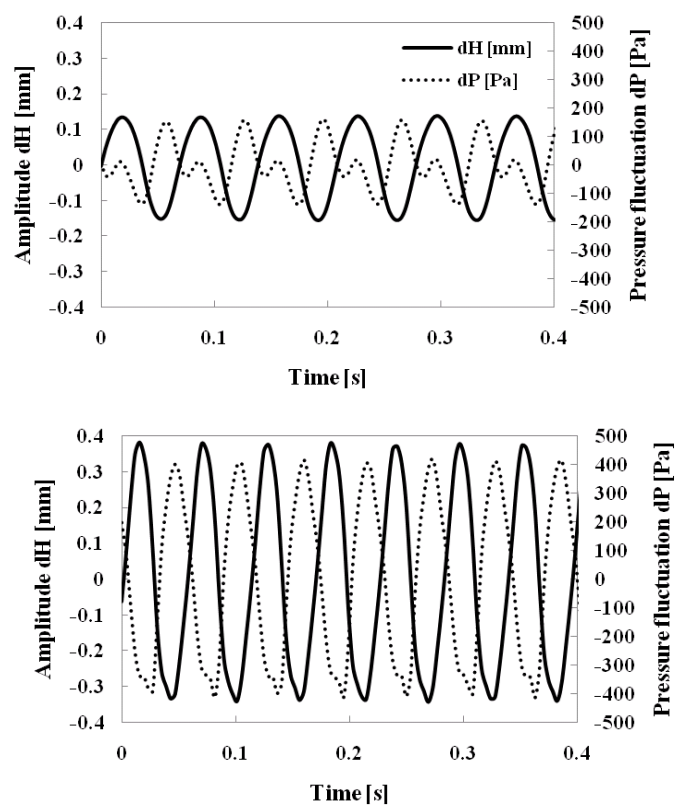

Fig.22 Time waveform of displacement and pressure fluctuation 

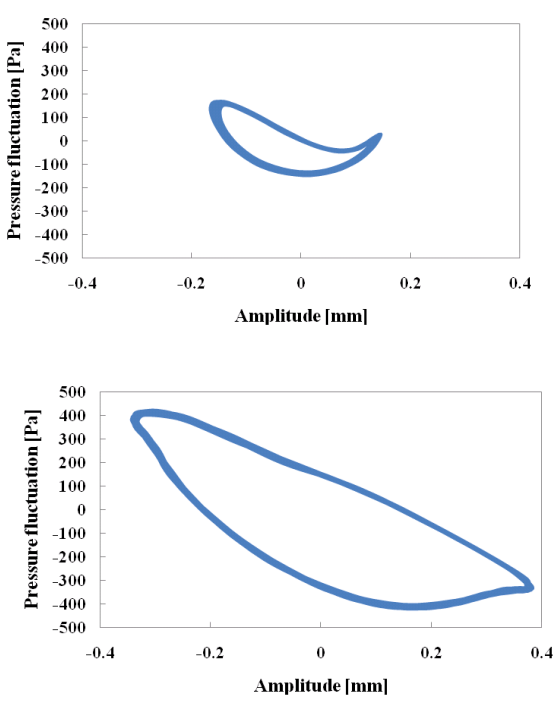

Fig.23 Lissajous Orbits

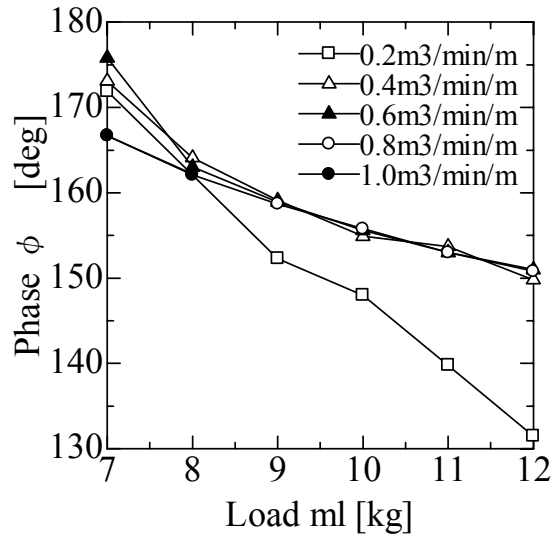

Fig.24 Phase of pressure relative to displacement

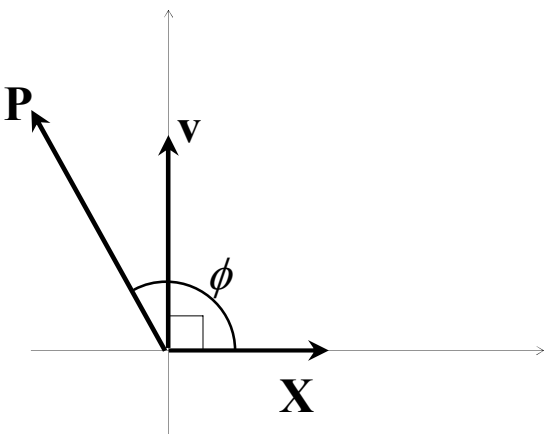

Fig.25 Phase of pressure relative to displacement and velocity

The belt displacement is positive when the belt moves downward. It can be seen that the amplitudes of the belt displacement and the pressure fluctuation becomes large when the flow rate increases. It can also be seen that the phase shift appears between the belt displacement and the pressure fluctuation. From the lissajous orbits it can be seen that the area enclosed by the orbits becomes large when the flow rate increases. This fact shows that 
the energy entering the vibrating system becomes large with an increasing flow rate.

Fig.24 shows the phase shift $\phi$ of the pressure fluctuation $\triangle P$ relative to the vibration displacement $\Delta H$ in the case of changing $Q$ and $m l$, where $m l$ is the concentrated load. It turns out that the phase shift $\phi$ becomes small with an increasing $m l$ and it was confirmed that the phase shift for each $m l$ becomes constant with $m l$ except in the case of $Q=0.2 \mathrm{~m}^{3} / \mathrm{min} / \mathrm{m}$.

Putting the displacement of the belt at $X=X_{0} \sin \omega_{n} t$, the velocity becomes

$$
\begin{aligned}
v & =\dot{X} \\
& =X_{0} \omega_{n} \cdot \cos \omega_{n} t \\
& =X_{0} \omega_{n} \cdot \sin \left(\omega_{n} t+\frac{\pi}{2}\right)
\end{aligned}
$$

where $\omega_{n}$ is a natural angular frequency.

Namely, the phase of the velocity presses forward $\pi / 2$ against the displacement. The power transferred to the belt can be expressed by

$$
\begin{aligned}
E & =\widetilde{P} \cdot \widetilde{v} \\
& =|\widetilde{P}||\widetilde{v}| \cos (\phi-\pi / 2)
\end{aligned}
$$

where $\widetilde{P}$ and $\widetilde{v}$ are the pressure and the velocity, respectively. Furthermore, they are complex values.

The phase relations among the pressure $\widetilde{P}$, the displacement $\widetilde{X}$, and the velocity $\widetilde{v}$ are shown in Fig.25. When the phase shift approaches $\pi / 2$, the power entering the system becomes large from Eq.(8).

\section{Conclusions}

Abnormal vibration has often occurred in a flow dynamic conveyer (FDC). An experimental apparatus was made, and the vibration of the belt and the pressure between the belt and trough were measured in order to clarify the mechanism. As a result, the following findings were obtained:

(1) The present theory may be effective and useful in obtaining the natural frequency of the FDC although the theory is still inadequate.

(2) The abnormal vibration does not occur in a parallel flow channel.

(3) The region where the abnormal vibration occurs becomes wide with an increasing load.

(4) The vibration amplitude becomes large and saturates with an increasing flow rate.

(5) The phase of the pressure relative to the displacement becomes smaller from $\pi$ to $\pi / 2$ with an increasing load and the values become the same independent of the flow rate. This is thought to be essential for the mechanism of abnormal vibration.

\section{References}

[1] F. INADA and S. HAYAMA, A Study on Leakage-Flow-Induced Vibrations ( $1^{\text {st }}$ Report, Fluid-Dynamic Forces Acting on the Walls of a One-Dimensional, Narrow, Tapered Passage), Transactions of the JSME, Vol.53, Vol.488, 1988, pp933-939. (in Japanese)

[2] F. INADA and S. HAYAMA, A Study on Leakage-Flow-Induced Vibrations ( $3^{\text {rd }}$ Report, Moment of Fluid-Dynamic Forces Acting on the Walls, One of which is Vibrating with Rotational Mode), Transactions of the JSME, Vol.54, Vol.507, 1988, pp.2565-2570. (in Japanese) 
[3] M.ARAI and K. TAJIMA, Leakage-Flow-Induced Vibrations of an Axisymmetric Body ( $1^{\text {st }}$ Report, Analysis of the Fluid Dynamic Forces Excited on an Axisymmetric Body), Transactions of the JSME, Vol.57, Vol.536, 1991, pp.1113-1121. (in Japanese)

[4] Dong-Wei LI, S. KANEKO and S. HAYAMA, A Study on the Annular Leakage-Flow-Induced Vibrations $\left(1^{\text {st }}\right.$ Report, Stability for Translational and Rotational Single-Degree-of-Freedom Systems), Transactions of the JSME, Vol.65, Vol.635, 1999, pp.2251-2256. (in Japanese)

[5] Dong-Wei LI, S. KANEKO and S. HAYAMA, A Study on the Annular Leakage-Flow-Induced Vibrations (2nd Report, Stability Analysis and Experiments for Translationally and Rotationally Coupled Two-Degree-of-Freedom Systems), Transactions of the JSME, Vol.65, Vol.635, 1999, pp.2257-2262. (in Japanese)

[6] N. OOMORI and M. KOBAYASHI, Stability Analysis of Flexible Belt floating on Air Flow, Paper of JSME, D\&D2000, No.342, CD, (in Japanese) [7] INCE/J Edition, Noise Countermeasure of Duct Sysyem, Gihodo Syuppan,1999

[7] N. OOMORI and M. KOBAYASHI, Stability Analysis of Flexible Belt floating on Air Flow ( $2^{\text {nd }}$ Report, In case of Loading), Paper of JSME, $D \& D 2000$, No.342, CD, (in Japanese)

[8] K. Ishihara, On Abnormal Vibration Generated in Flow Dynamic Conveyer ( Understanding of Phenomenon ), Transactions of the JSME, Vol.73, No. 729, Series C, 2007, pp.1279-1284. (in Japanese)

[9] K.Ishihara, On Abnormal Vibration Generated in Flow Dynamic Conveyer, Journal of System Design and Dynamics, Vol.2, No.2, 2008, pp.550-560 